\title{
Diabetic cardiac autonomic neuropathy: Effects of statins and omega-3 polyunsaturated fatty acids on lipid profile and insulin resistance parameters
}

\author{
Victoria A Serhiyenko ${ }^{1 *}$, Ludmila M Serhiyenko ${ }^{2}$, Samir Ajmi ${ }^{1}$ and Alexandr A Serhiyenko ${ }^{1}$ \\ ${ }^{1}$ Department of Endocrinology, Danylo Halytsky Lviv National Medical University, Lviv, Ukraine \\ ${ }^{2}$ Department of Medical Biology, Danylo Halytsky Lviv National Medical University, Lviv, Ukraine
}

\begin{abstract}
Background: Currently, there is no unified treatment algorithm of cardiac autonomic neuropathy (CAN) in patients with type 2 diabetes mellitus (T2DM). The aim of this study was to investigate the effects of simvastatin (SIM) and $\omega-3$ polyunsaturated fatty acids ( $\omega-3$ PUFAs) on blood lipid profile and insulin resistance (IR) in patients with T2DM and definite CAN.

Methods: The study involved 72 patients with T2DM and definite CAN. Patients were divided into four groups: 1st - received standard hypoglycemic therapy control ( $\mathrm{n}=15)$; 2nd ( $\mathrm{n}=22)$ - in addition SIM $20 \mathrm{mg} / \mathrm{q} . \mathrm{d}$.; 3rd ( $\mathrm{n}=18)$ - in addition 1 capsule/q.d. of the $\omega-3$ PUFAs; 4th $(\mathrm{n}=17)$ - in addition SIM $10 \mathrm{mg} / \mathrm{q} . \mathrm{d}$ and 1 capsule/q.d of the $\omega-3$ PUFAs for three months. The concentration of glucose, glycated hemoglobin A1c, immunoreactive insulin (IRI), total cholesterol (TC), low-density lipoprotein cholesterol (LDL-C), high-density lipoprotein cholesterol (HDL-C), triglycerides (TG) in the blood were determined. Homeostasis model assessment IR (HOMA-IR), atherogenic coefficient (AC), TG/LDL-C, TG/TC, TG/LDL-C and TG glucose (TyG) were calculated.

Results: Prescription of SIM was accompanied by a statistically significant decrease in TC, LDL-C, TG concentrations. In parallel, SIM induced a decrease of $\mathrm{AC}$, TG/HDL-C, increase in HDL-C, and does not affect the IRI, HOMA-IR, TG/LDL-C, TG/TC, TC/LDL-C/HDL-C, TyG. The use of $\omega-3$ PUFAs has contributed to a significant reduction in TG, AC, TG/LDL-C, TG/TC, TG/HDL-C, TyG index, increase in HDL-C, and was not accompanied by changes in IRI content, HOMA-IR, TC, LDL-C, and TC/LDL-C/HDL-C. The combined prescription of SIM and $\omega-3$ PUFAs was accompanied by more pronounced, statistically significant changes in the blood lipid spectrum, as well as a decrease in the IRI and HOMA-IR.
\end{abstract}

Conclusion: Obtained results justify the appropriateness of combined SIM and $\omega-3$ PUFAs prescription to patients with T2DM and definite CAN.

\begin{abstract}
Abbreviations: $\omega-3$ PUFAs $=\omega-3$ polyunsaturated fatty acids; $A C$ $=$ atherogenic coefficient CAN $=$ cardiac autonomic neuropathy; DLP = dyslipoproteinemia; HDL-C $=$ high-density lipoprotein cholesterol; HOMA-IR = homeostasis model assessment IR; IR = insulin resistance; IRI = immunoreactive insulin; LDL-C = low-density lipoprotein cholesterol; $\mathrm{SIM}=$ simvastatin; $\mathrm{T} 2 \mathrm{DM}=$ type 2 diabetes mellitus; $\mathrm{TC}=$ total cholesterol; $\mathrm{TG}=$ triglyceride; $\mathrm{TG} / \mathrm{HDL}-\mathrm{C}=$ triglyceride $/$ high-density lipoprotein cholesterol ratio; TG/LDL-C = triglyceride/ low-density lipoprotein cholesterol ratio; TG/LDL-C = triglyceride/ low-density lipoprotein cholesterol ratio; $\mathrm{TG} / \mathrm{TC}=$ triglyceride/total cholesterol ratio; $\mathrm{TyG}=\mathrm{TG}$ glucose index.
\end{abstract}

\section{Introduction}

The majority of patients with type 2 diabetes mellitus (T2DM) is diagnosed with coronary heart disease (CHD) due to coronary artery atherosclerosis. Metabolic alterations in the myocardium are combined with early coronary atherosclerosis. The development of diabetic cardiac autonomic neuropathy (CAN) is associated with the lesion of the autonomic nervous system and may be accompanied by coronary vessels ischemia, arrhythmias, "silent" myocardial infarction, severe orthostatic hypotension and sudden death syndrome [1,37]. Therefore, the problem of effective CAN treatment is particularly relevant. Pathogenetic treatment of CAN includes diet and physical activity; reducing of insulin resistance (IR); optimal glycemic control; treatment of dyslipoproteinemia (DLP); prevention and treatment of thrombosis; symptomatic treatment [2,7-10].

Classically, statins are used to manage DLP, but they also present beneficial effects beyond lipid-lowering; for instance, blood pressure (BP) reduction in central and peripheral sympathetic activity, and improvement in anti-inflammatory responses. A number of mechanisms have been proposed to explain the pleiotropic effect of statin therapy to reduce sympathetic outflow in cardiovascular diseases (CVD) [11-13]. Evidence from randomized clinical trials and metaanalysis supports statin treatment for the primary and secondary prevention of CVD [13].

Numerous studies report salutary effects of $\omega-3$ polyunsaturated fatty acids ( $\omega$-PUFAs) on CVD risk factors. These effects include

${ }^{*}$ Correspondence to: Victoria A Serhiyenko, Assistant Professor, Department of Endocrinology, Danylo Halytsky Lviv National Medical University, 69, Pekarska Str., 79010, Lviv, Ukraine, Tel: 00380987463315; E-mail: serhiyenkoa@gmail. com; serhiyenkov@gmail.com

Key words: diabetes mellitus, cardiac autonomic neuropathy, treatment

Received: September 09, 2019; Accepted: September 17, 2019; Published: September 20, 2019 

parameters

lowering of triglyceride (TG); lowering of BP; decreasing platelet aggregation and inflammation; protection from arrhythmias. Systematic meta-analysis suggests that high doses of $\omega$-3 PUFAs $(\sim 3$ $\mathrm{g}$ /day) produce a small, but significant decrease in systolic BP in older and hypertensive subjects $[9,14]$.

The aim of this study was to investigate the effects of simvastatin and $\omega$-PUFAs on blood lipid profile and IR in patients with T2DM and definite CAN.

\section{Materials and Method}

This was an open-label comparative controlled randomized study. Study inclusion criteria: age: 45-60 years old; T2DM with optimal or suboptimal glycemic control; T2DM patients with confirmed CAN; clinical stages of diabetic polyneuropathy; BMI within $20-30 \mathrm{~kg} / \mathrm{m}^{2}$; consent to observe the dietary regime with the limited use of foods containing saturated fatty acids during the study; consent to maintain appropriate physical activity. The examination excluded persons with uncontrolled DM, ketosis, peripheral vascular diseases, ischemic form of diabetic foot, distal neuropathy caused by other reasons than diabetes (neurological disease, chronic alcoholism, pharmacological agents), neoplasms, hypothyroidism, history of acute pancreatitis, affected activity of lipoprotein lipase and/or DLP type III, women during pregnancy and lactation, patients with T1DM, hypersensitivity to the components of the medicinal product. Treatment with antidepressants, anticonvulsants, opiates, capsaicin, neuroleptic agents, cytostatic agents, B group vitamins, benfotiamine, $\gamma$-linoleic acid, aldose reductase inhibitors, isotretinoin (accutane), warfarin, antioxidants (including $\alpha$-lipoic acid medications), $\omega-3$ PUFAs for six months prior to the inclusion in the study was also exclusion criteria. Patients suffering from severe diseases of the heart, lungs, liver, pancreas, and intestines were also excluded from the study.

The study has been conducted for 2 years. Each patient examined before the beginning of the study did not take $\omega-3$ PUFAs and was on a stable regime of hypoglycemic and antihypertensive treatment for 6 months. After CAN diagnosing simvastatin (SIM) and/or $\omega$-3 PUFAs was prescribed to patients from the treatment groups according to the study design. The treatment of the control group was unchanged. The duration of the treatment period was 3 months. To determine the metabolic effects of the treatment the glycemic, lipids and IR parameters were performed initially and after the end of treatment.

All patients were residents of the city of Lviv observed by an endocrinologist at the Department of Endocrinology of the Danylo Halitsky Lviv National Medical University based on Lviv Regional State Clinical Treatment and Diagnostical Endocrinological Center. On the basis of these institutions, patients were examined and treated.
Examination plan included determination of glucose, glycated hemoglobin Alc (HbAlc), lipids, IR parameters in the blood, Electrocardiography (ECG), five cardiovascular autonomic reflex tests (CARTs) and Holter-ECG. All patients underwent screening for CAN that included five CARTs, in addition, time-domain and frequencydomain heart rate variability tests were performed $[1,3,15]$.

The study involved 72 patients with T2DM and definite CAN. Clinical characteristics of studied patients with T2DM and definite CAN are given in Table 1. All participants signed an informed consent prior to their inclusion in the study. Patients were divided into four treatment groups: 1st group - received standard hypoglycemic therapy - control ( $\mathrm{n}=15) ; 2$ 2nd group $(\mathrm{n}=22)$ - standard standard hypoglycemic therapy and SIM $20 \mathrm{mg} / \mathrm{q} . \mathrm{d}$.; 3rd group (standard hypoglycemic therapy and 1 capsule/q.d. of the $\omega$-3 PUFAs ( $1 \mathrm{~g}$, including $90 \%$ $\omega$-3 PUFAs, mainly eicosapentaenoic (EPA) and docosahexaenoic acid (DHA) and $4 \mathrm{mg}$ of $\alpha$-tocopherol acetate); 4 th - standard hypoglycemic therapy, SIM $10 \mathrm{mg} / \mathrm{q} . d$. and 1 capsule/q.d. of the $\omega$-3 PUFAs for three months. The standard hypoglycemic treatment of DM included dietary regime, appropriate physical activity, and oral antihyperglycemic drugs. The therapy of the control group remained unchanged during the treatment period.

The concentration of glucose in the blood was determined by the glucose oxidase method while HbAlc level was assessed by using a highly sensitive method of ion-exchange liquid chromatography with D-10 analyzer and BIO-RAD reagents (United States). Determination of immunoreactive insulin (IRI) was performed using commercial kits from Immunotech insulin immunoradiometric assay reagents (Czech Republic). Lipid metabolism was assessed by the concentration of total cholesterol (TC), TG, low-density lipoprotein (LDL-C), high-density lipoprotein (HDL-C); atherogenic coefficient (AC) and AC, TG/ LDL-C, TG/TC, TG/LDL-C, TG/HDL-C parameters. The TG-glucose (TyG) index was calculated by the Ln [fasting TG (mg/dL) x fasting glucose $(\mathrm{mg} / \mathrm{dL}) / 2$ ] [16]. The use of the homeostasis model assessment (HOMA) IR (HOMA-IR) [17], the insulin suppression test [18], and the hyperinsulinemic-euglycemic clamp suggested that the TyG index correlated with IR [17]. The lipid fractions were determined by using HUMAN reagents (Germany) for the analyzer Humanlayzer 2000. HOMA-IR index was calculated according to the formula: fasting IRI $(\mathrm{mcIU} / \mathrm{mL}) \mathrm{x}$ fasting glucose $(\mathrm{mmol} / \mathrm{L}) / 22.5$ [19].

Resting 12-lead surface electrocardiography (ECG) with a paper speed of $25 \mathrm{~mm} / \mathrm{s}$ and a signal size of $10 \mathrm{~mm} / \mathrm{mV}$ was recorded in the morning period. We performed resting ECG analysis included measurement of the following parameters: heart rhythm, heart rate, conduction intervals, and Holter-ECG [(ECG "EC-3H" ("Labtech," Hungary)] analysis included measurement of 24 hours ECG, circadian indexes and heart rate variability parameters.

Table 1. Baseline characteristics of patients included in this study

\begin{tabular}{|c|c|c|c|c|}
\hline \multirow[t]{2}{*}{ Parameter } & \multicolumn{4}{|c|}{ Patients with T2DM and definite CAN $(n=72)$} \\
\hline & Control $(n=15)$ & $\operatorname{SIM}(n=22)$ & $\omega-3$ PUFAs $(n=18)$ & SIM + $\Omega-3$ PUFAs $(n=17)$ \\
\hline & Group 1 & Group 2 & Group 3 & Group 4 \\
\hline Age, years & $55.33 \pm 0.95$ & $54.14 \pm 0.89$ & $54.83 \pm 0.87$ & $52.35 \pm 1.12$ \\
\hline DM duration, yrs & $3.6 \pm 0.42$ & $4.68 \pm 0.62$ & $3.44 \pm 0.42$ & $4.82 \pm 0.84$ \\
\hline BMI, $\mathrm{kg} / \mathrm{m}^{2}$ & $28.89 \pm 0.16$ & $28.5 \pm 0.39$ & $28.28 \pm 0.33$ & $28.91 \pm 0.19$ \\
\hline $\mathrm{SBP}, \mathrm{mm} \mathrm{Hg}$ & $143.0 \pm 3.06$ & $149.5 \pm 3.15$ & $146.6 \pm 2.19$ & $152.12 \pm 3.74$ \\
\hline $\mathrm{DBP}, \mathrm{mm} \mathrm{Hg}$ & $90.6 \pm 1.84$ & $91.14 \pm 2.12$ & $92.72 \pm 1.86$ & $93.06 \pm 2.22$ \\
\hline $\mathrm{PP}, \mathrm{mm} \mathrm{Hg}$ & $52.4 \pm 2.04$ & $58.36 \pm 2.83$ & $97.78 \pm 3.25$ & $58.47 \pm 2.83$ \\
\hline HR, bpm & $95.2 \pm 2.81$ & $90.55 \pm 2.31$ & $97.78 \pm 3.25$ & $89.88 \pm 2.41$ \\
\hline
\end{tabular}

T2DM = type 2 diabetes mellitus; CAN = cardiac autonomic neuropathy; DM = diabetes mellitus; BMI = body mass index; SIM = simvastatin; $\omega-3$ PUFAs = $\omega-3$ polyunsaturated fatty acids; $\mathrm{SBP}=$ systolic blood pressure; $\mathrm{DBP}=$ diastolic blood pressure; $\mathrm{PP}=$ pulse pressure; $\mathrm{HR}=$ heart rate, $\mathrm{bpm}=$ beats per minute. 
Serhiyenko VA (2019) Diabetic cardiac autonomic neuropathy: Effects of statins and omega-3 polyunsaturated fatty acids on lipid profile and insulin resistance parameters

The work was done according to the principles of the Declaration of Helsinki (2004) and was approved by an Ethics Committee of the Danylo Halytsky Lviv Medical University, protocol № 2 from 18 February 2013. All subjects signed informed consent prior to their inclusion in the study. The research performed corresponded to the generally accepted norms of morality and observance of the rights, interests and personal dignity of the persons participating in the study.

Statistical analysis was based on the variational method using a statistical parametric t-test, nonparametric Wilcoxon t-test, and Fisher's Pearson correlation coefficient. Data are presented as mean \pm standard error of the mean (SEM). All tests were performed using the ANOVA (MicroCal Origin v. 8.0) software. Statistical significance was set at $\mathrm{p}<0.05$.

\section{Results}

We found out that the baseline level of HbA1c, HOMA-IR parameters, and the blood lipids profile after three months do not present statistically significant differences in the control group. Changes of HbAlc, IRI and HOMA-IR parameters among patients with T2DM and definite CAN after 3-months of SIM, $\omega$-PUFAs, and combined SIM plus $\omega$-PUFAs therapy are given in Table 2.

It was established that SIM and $\omega-3$ PUFAs therapy do not contribute to statistically significant changes in such parameters as HbAlc, IRI, and HOMA-IR parameters. The combined prescription of SIM and $\omega-3$ PUFAs was accompanied by a statistically significant decrease in the level of IRI and HOMA-IR parameters.

Changes of some lipid parameters and lipid ratios in patients with T2DM and definite CAN after 3-months of SIM, $\omega$-PUFAs, and combined SIM plus $\omega$-PUFAs therapy are given in Tables 3 and 4 .

Obtained results of our study could testify that the prescription of SIM was accompanied by a statistically significant decrease in TC $(\mathrm{p}<$ $0.001)$, LDL-C ( $<<0.001)$, TG concentrations $(\mathrm{p}<0.05)$. In parallel, SIM induced an decrease of AC ( $\mathrm{p}<0.01)$, TG/HDL-C $(\mathrm{p}<0.05)$, and increase in HDL-C levels $(\mathrm{p}<0.05)$, and does not affect the TG/ LDL-C, TG/TC, TC/LDL-C/HDL-C, TyG index parameters. The use of $\omega-3$ PUFAs has contributed to a significant reduction in TG $(\mathrm{p}<$ $0.001)$, AC $(\mathrm{p}<0.05)$, TG/LDL-C $(\mathrm{p}<0.001)$, TG/TC $(\mathrm{p}<0.001)$, TG/HDL-C $(\mathrm{p}<0.001)$, TyG index $(\mathrm{p}<0.001)$, increase in HDL-C

Table 2. Changes of the HbA1c, IRI, and HOMA-IR in patients with T2DM and definite CAN after 3-months of SIM and $\omega$-3 PUFAs therapy

\begin{tabular}{|c|c|c|c|c|}
\hline \multirow[t]{2}{*}{ Parameter } & \multicolumn{4}{|c|}{ Patients with T2DM and definite CAN $(\mathrm{n}=72)$} \\
\hline & Groups & Baseline & After treatment & $\%$ change \\
\hline \multirow{4}{*}{$\mathrm{HbA} 1 \mathrm{c}, \%$} & Control $(n=15)$ & $7.17 \pm 0.18$ & $7.21 \pm 0.19$ & $+0.6 \pm 1.07$ \\
\hline & $\operatorname{SIM}(\mathrm{n}=22)$ & $7.4 \pm 0.23$ & $7.34 \pm 0.19$ & $-1.18 \pm 1.25$ \\
\hline & $\Omega$-3 PUFAs $(\mathrm{n}=18)$ & $7.03 \pm 0.17$ & $7.07 \pm 0.14$ & $+0.87 \pm 1.22$ \\
\hline & $\operatorname{SIM}+\Omega-3$ PUFAs $(\mathrm{n}=17)$ & $6.96 \pm 0.22$ & $6.88 \pm 0.22$ & $-1.04 \pm 1.33$ \\
\hline \multirow{4}{*}{ IRI, $\mu \mathrm{IU} / \mathrm{mL}$} & Control $(n=15)$ & $27.39 \pm 2.13$ & $26.01 \pm 2.25$ & $-6.43 \pm 3.0$ \\
\hline & $\operatorname{SIM}(\mathrm{n}=22)$ & $29.53 \pm 1.99$ & $26.16 \pm 1.6$ & $-8.91 \pm 4.21$ \\
\hline & $\Omega$-3 PUFAs $(\mathrm{n}=18)$ & $25.07 \pm 2.65$ & $23.44 \pm 2.34$ & $-4.23 \pm 5.62$ \\
\hline & $\operatorname{SIM}+\Omega-3$ PUFAs $(\mathrm{n}=17)$ & $26.37 \pm 1.99$ & $20.24 \pm 1.09^{*}$ & $-21.07 \pm 2.05$ \\
\hline \multirow{4}{*}{ HOMA-IR } & Control $(\mathrm{n}=15)$ & $9.04 \pm 0.99$ & $8.46 \pm 0.99$ & $-6.12 \pm 4.06$ \\
\hline & $\operatorname{SIM}(\mathrm{n}=22)$ & $8.99 \pm 0.94$ & $7.43 \pm 0.6$ & $-8.86 \pm 6.84$ \\
\hline & $\Omega$-3 PUFAs $(\mathrm{n}=18)$ & $7.9 \pm 1.06$ & $7.29 \pm 0.86$ & $-8.86 \pm 6.84$ \\
\hline & $\operatorname{SIM}+\Omega-3$ PUFAs $(\mathrm{n}=17)$ & $7.4 \pm 0.92$ & $5.12 \pm 0.44^{*}$ & $-25.35 \pm 3.16$ \\
\hline
\end{tabular}

Data are presented as absolute values and as $\%$ change from baseline $(\Delta \%$, Mean $\pm \mathrm{SEM}) ; \mathrm{p}=$ value $=\mathrm{p}<0.05^{*}$ compared to baseline; T2DM $=$ type 2 diabetes mellitus; CAN $=$ cardiac autonomic neuropathy; SIM = simvastatin; $\omega-3$ PUFAs $=\omega-3$ polyunsaturated fatty acids; HbA1c $=$ glycated hemoglobin A1c; IRI = immunoreactive insulin; IR = insulin resistance; HOMA-IR $=$ homeostasis model assessment IR.

Table 3. Changes of some lipid parameters in patients with T2DM and definite CAN after 3-months of SIM and $\omega$-3 PUFAs therapy

\begin{tabular}{|c|c|c|c|c|}
\hline \multirow[t]{2}{*}{ Parameter } & \multicolumn{3}{|c|}{ Patients with T2DM and definite CAN $(\mathrm{n}=72)$} & \multirow[b]{2}{*}{$\%$ change } \\
\hline & Groups & Baseline & After treatment & \\
\hline \multirow{4}{*}{$\mathrm{TC}, \mathrm{mmol} / \mathrm{L}$} & Control $(\mathrm{n}=15)$ & $6.59 \pm 0.18$ & $6.13 \pm 0.15$ & $-6.73 \pm 1.09$ \\
\hline & $\operatorname{SIM}(\mathrm{n}=22)$ & $6.18 \pm 0.29$ & $4.81 \pm 0.23^{\S \S}$ & $-21.83 \pm 1.55$ \\
\hline & $\Omega-3$ PUFAs $(\mathrm{n}=18)$ & $6.0 \pm 0.2$ & $5.64 \pm 0.24$ & $-5.52 \pm 3.16$ \\
\hline & $\operatorname{SIM}+\Omega-3$ PUFAs $(n=17)$ & $6.41 \pm 0.13$ & $4.56 \pm 0.15^{8 \S}$ & $-28.69 \pm 1.99$ \\
\hline \multirow{4}{*}{ LDL-C, mmol/L } & Control $(\mathrm{n}=15)$ & $4.59 \pm 0.16$ & $4.25 \pm 0.17$ & $-8.27 \pm 1.44$ \\
\hline & $\operatorname{SIM}(\mathrm{n}=22)$ & $4.27 \pm 0.27$ & $2.72 \pm 0.14^{\S \S}$ & $-34.23 \pm 2.55$ \\
\hline & $\Omega-3$ PUFAs $(\mathrm{n}=18)$ & $4.09 \pm 0.18$ & $3.78 \pm 0.27$ & $-8.08 \pm 5.58$ \\
\hline & $\operatorname{SIM}+\Omega-3$ PUFAs $(\mathrm{n}=17)$ & $4.42 \pm 0.12$ & $2.57 \pm 0.11^{\S \S}$ & $-41.58 \pm 2.26$ \\
\hline \multirow{4}{*}{$\mathrm{HDL}-\mathrm{C}, \mathrm{mmol} / \mathrm{L}$} & Control $(\mathrm{n}=15)$ & $0.84 \pm 0.03$ & $0.87 \pm 0.03$ & $+4.09 \pm 0.97$ \\
\hline & $\operatorname{SIM}(\mathrm{n}=22)$ & $0.77 \pm 0.03$ & $0.92 \pm 0.06^{*}$ & $+12.1 \pm 2.32$ \\
\hline & $\Omega-3$ PUFAs $(\mathrm{n}=18)$ & $0.78 \pm 0.03$ & $0.9 \pm 0.05^{*}$ & $+9.73 \pm 2.57$ \\
\hline & $\operatorname{SIM}+\Omega-3$ PUFAs $(\mathrm{n}=17)$ & $0.76 \pm 0.03$ & $0.89 \pm 0.04^{*}$ & $+16.51 \pm 1.16$ \\
\hline \multirow{4}{*}{$\mathrm{TG}, \mathrm{mmol} / \mathrm{L}$} & Control $(\mathrm{n}=15)$ & $2.52 \pm 0.12$ & $2.31 \pm 0.11$ & $-8.28 \pm 1.17$ \\
\hline & $\operatorname{SIM}(n=22)$ & $2.5 \pm 0.27$ & $1.83 \pm 0.18^{*}$ & $-22.57 \pm 1.2$ \\
\hline & $\Omega-3$ PUFAs $(\mathrm{n}=18)$ & $2.47 \pm 0.15$ & $1.62 \pm 0.09^{\$ \S}$ & $-33.35 \pm 2.73$ \\
\hline & $\operatorname{SIM}+\Omega-3$ PUFAs $(\mathrm{n}=17)$ & $2.75 \pm 0.15$ & $1.57 \pm 0.15^{\S \S}$ & $-43.28 \pm 2.91$ \\
\hline
\end{tabular}

Data are presented as absolute values and as $\%$ change from baseline $(\Delta \%$, Mean \pm SEM $) ; \mathrm{p}=$ value $=\mathrm{p}<0.05^{*}$ compared to baseline; $\mathrm{p}<0.01^{\S}$ compared to baseline; $\mathrm{p}<0.001^{\S \S}$ compared to baseline; T2DM = type 2 diabetes mellitus; $\mathrm{CAN}=$ cardiac autonomic neuropathy; $\mathrm{SIM}=$ simvastatin; $\omega-3$ PUFAs $=\omega-3$ polyunsaturated fatty acids; $\mathrm{TC}=$ total cholesterol; $\mathrm{LDL}-\mathrm{C}=$ low-density lipoprotein cholesterol; HDL-C = high-density lipoprotein cholesterol; TG = triglyceride. 
Serhiyenko VA (2019) Diabetic cardiac autonomic neuropathy: Effects of statins and omega-3 polyunsaturated fatty acids on lipid profile and insulin resistance parameters

Table 4. Changes of some lipid ratios in patients with T2DM and definite CAN after 3-months of SIM and $\omega$-3 PUFAs therapy

\begin{tabular}{|c|c|c|c|c|}
\hline \multirow[t]{2}{*}{ Parameter } & \multicolumn{3}{|c|}{ Patients with T2DM and definite CAN $(\mathrm{n}=72)$} & \multirow[b]{2}{*}{$\%$ change } \\
\hline & Groups & Baseline & After treatment & \\
\hline \multirow{4}{*}{$\mathrm{AC}$} & Control $(\mathrm{n}=15)$ & $7.05 \pm 0.43$ & $6.2 \pm 0.37$ & $-11.79 \pm 1.38$ \\
\hline & $\operatorname{SIM}(\mathrm{n}=22)$ & $7.4 \pm 0.59$ & $4.73 \pm 0.48^{\S}$ & $-37.16 \pm 2.51$ \\
\hline & $\Omega$-3 PUFAs $(\mathrm{n}=18)$ & $6.9 \pm 0.47$ & $5.6 \pm 0.44^{*}$ & $-20.17 \pm 7.24$ \\
\hline & SIM $+\Omega-3$ PUFAs $(n=17)$ & $7.56 \pm 0.54$ & $4.25 \pm 0.32^{\S \S}$ & $-43.54 \pm 2.39$ \\
\hline \multirow{4}{*}{ TG/LDL-C } & Control $(\mathrm{n}=15)$ & $0.55 \pm 0.02$ & $0.54 \pm 0.03$ & $-0.39 \pm 2.06$ \\
\hline & $\operatorname{SIM}(\mathrm{n}=22)$ & $0.64 \pm 0.06$ & $0.71 \pm 0.08$ & $+18.15 \pm 5.57$ \\
\hline & $\Omega-3$ PUFAs $(\mathrm{n}=18)$ & $0.61 \pm 0.03$ & $0.46 \pm 0.03^{\S \S}$ & $-22.2 \pm 5.61$ \\
\hline & $\operatorname{SIM}+\Omega-3$ PUFAs $(\mathrm{n}=17)$ & $0.64 \pm 0.06$ & $0.65 \pm 0.08$ & $-0.03 \pm 7.41$ \\
\hline \multirow{4}{*}{ TG/TC } & Control $(\mathrm{n}=15)$ & $0.38 \pm 0.01$ & $0.37 \pm 0.02$ & $-1.07 \pm 1.8$ \\
\hline & $\operatorname{SIM}(\mathrm{n}=22)$ & $0.4 \pm 0.04$ & $0.38 \pm 0.03$ & $-0.12 \pm 4.08$ \\
\hline & $\Omega-3$ PUFAs $(\mathrm{n}=18)$ & $0.41 \pm 0.016$ & $0.29 \pm 0.01^{\S \S}$ & $-28.34 \pm 3.47$ \\
\hline & $\operatorname{SIM}+\Omega-3$ PUFAs $(\mathrm{n}=17)$ & $0.43 \pm 0.03$ & $0.35 \pm 0.04$ & $-19.3 \pm 4.54$ \\
\hline \multirow{4}{*}{ TG/HDL-C } & Control $(\mathrm{n}=15)$ & $3.09 \pm 0.21$ & $2.74 \pm 0.2$ & $-11.65 \pm 1.58$ \\
\hline & $\operatorname{SIM}(\mathrm{n}=22)$ & $3.46 \pm 0.43$ & $2.21 \pm 0.28^{*}$ & $-33.1 \pm 3.06$ \\
\hline & $\Omega$-3 PUFAs $(\mathrm{n}=18)$ & $3.29 \pm 0.27$ & $1.89 \pm 0.15^{\S \S}$ & $-41.22 \pm 2.24$ \\
\hline & $\operatorname{SIM}+\Omega-3$ PUFAs $(\mathrm{n}=17)$ & $3.78 \pm 0.35$ & $1.83 \pm 0.18^{\S \S}$ & $-51.26 \pm 2.68$ \\
\hline \multirow{4}{*}{ TC/LDL-C/HDL-C } & Control $(\mathrm{n}=15)$ & $1.75 \pm 0.06$ & $1.7 \pm 0.07$ & $-2.74 \pm 1.74$ \\
\hline & $\operatorname{SIM}(\mathrm{n}=22)$ & $2.03 \pm 0.12$ & $2.17 \pm 0.17$ & $+5.89 \pm 5.02$ \\
\hline & $\Omega$-3 PUFAs $(\mathrm{n}=18)$ & $1.91 \pm 0.05$ & $1.77 \pm 0.06$ & $-6.87 \pm 3.08$ \\
\hline & SIM $+\Omega-3$ PUFAs $(\mathrm{n}=17)$ & $1.99 \pm 0.11$ & $2.1 \pm 0.12$ & $+6.9 \pm 4.19$ \\
\hline \multirow{4}{*}{ TyG index } & Control $(\mathrm{n}=15)$ & $9.53 \pm 0.06$ & $9.44 \pm 0.05$ & $-0.89 \pm 0.39$ \\
\hline & $\operatorname{SIM}(\mathrm{n}=22)$ & $9.27 \pm 0.14$ & $8.96 \pm 0.13$ & $-3.29 \pm 0.3$ \\
\hline & $\Omega$-3 PUFAs $(\mathrm{n}=18)$ & $9.46 \pm 0.08$ & $9.05 \pm 0.08^{\S \S}$ & $-4.24 \pm 0.68$ \\
\hline & $\operatorname{SIM}+\Omega-3$ PUFAs $(\mathrm{n}=17)$ & $9.41 \pm 0.09$ & $8.76 \pm 0.12^{\S \S}$ & $-6.94 \pm 0.61$ \\
\hline
\end{tabular}

Data are presented as absolute values and as $\%$ change from baseline $(\Delta \%$, Mean \pm SEM $) ; \mathrm{p}=$ value $=\mathrm{p}<0.05^{*}$ compared to baseline; $\mathrm{p}<0.01^{\S}$ compared to baseline; $\mathrm{p}<0.001^{\S \S}$ compared to baseline; T2DM = type 2 diabetes mellitus; $\mathrm{CAN}=$ cardiac autonomic neuropathy; $\mathrm{SIM}=$ simvastatin; $\omega-3$ PUFAs $=\omega-3$ polyunsaturated fatty acids; $\mathrm{AC}=$ atherogenic coefficient; TG/ LDL-C = triglyceride/low-density lipoprotein cholesterol ratio; TG/TC = triglyceride/total cholesterol ratio; TG/LDL-C = triglyceride/low-density lipoprotein cholesterol ratio; TG/HDL-C $=$ triglyceride/high-density lipoprotein cholesterol ratio; TyG $=$ TG glucose index .

levels ( $\mathrm{p}<0.05)$, and was not accompanied by statistically significant changes in TC, LDL-C, and TC/LDL-C/HDL-C index parameters. The combined prescription of SIM and $\omega-3$ PUFAs was accompanied by more pronounced, statistically significant changes in the blood lipid spectrum, as well as a decrease in the level of IRI $(\mathrm{p}<0.05)$, and HOMA-IR parameters $(\mathrm{p}<0.05)$.

\section{Discussion}

Type 2 diabetes mellitus is known to be characterized by the presence of hypertriglyceridemia, one of the key components of diabetic DLP, which significantly increases the risk of CVD development. Statin therapy is considered as the cornerstone of clinician's efforts toward primary and secondary CVD prevention in patients with T2DM [20,21]. Patients with T2DM are deemed as prime candidates for receiving statin therapy, which has been endorsed by most of the clinical practice guidelines [22]. In accordance with what has been recommended earlier by American College of Cardiology/American Heart Association guidelines, American Diabetes Association standards of care recommend moderate-intensity statins for all T2DM patients over the age of 40 years as a primary prophylaxis [22] On the other hand, higher doses of statins are required for the secondary prophylaxis of diabetic patients with coronary artery disease (CAD) or at increased CVD risks such as those with abnormal LDL-C levels, smokers, hypertension, or albuminuria [22]. Adherence to clinical guidelines that recommend statins for T2DM patients as the main CVD prophylaxis treatment is modulated by many factors. These determinants may play a crucial role in ensuring success following the decision to prescribe statins. Moreover, having prescribed statins, some factors related to both clinicians and patients alike can also affect compliance with statin therapy. Initiatives to enhance statin therapy prescribing should recognize the comprehensive nature of the prescribing process. Efforts to assure proper statin utilization and prescribing may help in achieving better clinical outcomes of statin therapy among patients with T2DM [13].

There are quite a number of conflicting reports regarding the potential positive effects of $\omega-3$ PUFAs in patients with T2DM and CVD. In particular, the recently published results of several metaanalyses indicate that $\omega \mathrm{a}-3$ PUFAs are not capable of reducing the risk of cardiovascular events [23]. No benefits of taking $\omega$-3 PUFAs in patients with T2DM and atherosclerotic vascular disease, in particular, no significant effects on oxidative stress, chronic low-intensity inflammatory process parameters, coagulation status and metabolic status have been found out [24]. In contrast, the results of a large meta-analysis of randomized controlled trials have shown that the addition of $\omega$-3 PUFAs has a favorable lipid-lowering effect, reduces the level of pro-inflammatory cytokines and improves glycemia $[25,26]$. The positive findings of this meta-analysis are complemented by the results of a number of recent studies that have demonstrated that $\omega-3$ PUFAs have beneficial effects on metabolism in patients with T2DM [27-29]. In patients with impaired glucose metabolism and CAD, EPA has been reported to contribute to the correction of postprandial hypertriglyceridemia, hyperglycemia, insulin production, endothelial dysfunction [27]. Omega-3 PUFAs have been reported to have a positive effect on glucose, $\mathrm{Hb} 1 \mathrm{Ac}$, leptin and leptin/adiponectin ratios in patients with T2DM [28]. The conflicting results of different original studies and a meta-analysis can be partially explained by the different dosage and duration of supplementation, each of which can modify the effects of $\omega$-3 PUFAs on cardio-metabolic biomarkers [30]. Meta-analyses are usually limited by the inability to draw conclusions regarding the dosage, duration, and interaction of the dosage, 

parameters

and duration of administration of $\omega-3$ PUFAs. However, almost all endpoints in the so-called "negative" meta-analyses tended to benefit, with an almost $10 \%$ decrease in cardiovascular outcomes and borderline statistical significance. In many studies included in these meta-analyses, an insufficient daily dose of $\omega-3$ PUFAs of less than $1000 \mathrm{mg}$ was tested [31].

Therefore, dietary consumption of $\omega-3$ PUFAs is recommended in international guidelines for the general population to prevent the occurrence of CHD. However, the precise mechanisms underlying the cardioprotective effects of $\omega-3$ PUFAs are not fully understood. Omega-3 PUFAs can be incorporated into the phospholipid bilayer of cell membranes and can affect membrane fluidity, lipid microdomain formation, and signaling across membranes. Omega-3 PUFAs also modulate the function of membrane ion channels, such as $\mathrm{Na}^{+}$and L-type $\mathrm{Ca}^{2+}$ channels, to prevent lethal arrhythmias. Moreover, $\omega-3$ PUFAs also prevent the conversion of arachidonic acid into proinflammatory eicosanoids by serving as an alternative substrate for cyclooxygenase or lipoxygenase pathways, resulting in the production of less potent products. In addition, a number of enzymatically oxygenated metabolites derived from $\omega-3$ PUFAs were recently identified as anti-inflammatory mediators. These $\omega-3$ metabolites may contribute to the beneficial effects against CHD that are attributed to $\omega-3$ PUFAs [32,33]. The OMEGA study including $94.2 \%$ statin users failed to prevent cardiovascular events [34]. The ORIGIN trial including $53.8 \%$ statin users also failed [34]. In the previous two studies (GISSI-P and GISSI-HF), EPA + DHA may be effective to prevent cardiovascular events because of fewer combined use of statins. In the recent studies (OMEGA and ORIGIN) which included many participants who had taken statins, EPA + DHA failed to reduce cardiovascular events. The combination of $\omega-3$ PUFAs and rosuvastatin in patients with residual hypertriglyceridemia, despite treatment with statins, led to a greater decrease in TG and non-HDL cholesterol than rosuvastatinmonotherapy [29].

However, the present study has several limitations. The duration of treatment was short. Additional long-term studies on the efficacy and tolerability of $\omega-3$ fatty acids are needed.

\section{Conclusion}

Our results suggest that the efficacy of SIM and $\omega-3$ PUFAs is not associated with improved glycemic control of T2DM in patients with definite CAN but is rather the result of a direct effect of the pharmacological agent on the investigated metabolic indexes. Therefore, the appointment of combined statins and $\omega-3$ PUFAs is necessary for the treatment of DLP in patients with T2DM and definite CAN. However, existing data are not consistent perhaps due to a significant heterogeneity (variable doses of statins and $\omega-3$ PUFAs, different duration of intake, different populations, and end-points) in the interventional studies. For prevention/treatment of CAN events supplementation with statins and $\omega-3$ PUFAs should be integrated into a more global strategy that includes focusing on other components of a healthy lifestyle (diet, weight control, physical activity, smoking cessation) and on tight control of glucose and lipid profile when indicated. Thus, further research to understand the mechanism of action and confirmation the beneficial effects of combined statins and $\omega-3$ PUFAs on the heart rate variability and artery stiffness parameters is needed.

\section{Author's contribution}

Victoria Serhiyenko, Ludmila Serhiyenko, Samir Ajmi, and Alexandr Serhiyenko contributed substantially to all aspects of this manuscript, including conception and design; acquisition, analysis, and interpretation of data; and drafting the article. Victoria Serhiyenko and Alexander Serhiyenko contributed substantially to the conception of this manuscript; and acquisition and analysis of data. All authors approved the final version of this article.

\section{Acknowledgements}

The authors would like to thank Veronika Segin for here assistance with data analysis.

\section{Funding}

This research did not receive any specific grant funding, neither from public agencies, commercial, nor from not-for-profit entities.

\section{Conflicts of interest}

There are no ethical/legal conflicts involved in the article. There are no conflicts of interest, including specific financial interests and relationships and affiliations relevant to the subject of their manuscript.

\section{References}

1. Spallone V, Ziegler D, Freeman R, Bernardi L, Frontoni S, et al. (2011) Toronto Consensus Panel on Diabetic Neuropathy. Cardiovascular autonomic neuropathy in diabetes: clinical impact, assessment, diagnosis, and management. Diabetes Metab Res Rev 27: 639-653.

2. Serhiyenko V, Serhiyenko A (2018) Diabetic cardiovascular neuropathy. Stavropol: Logos Publishers.

3. Vinik AI, Erbas T, Casellini CM (2013) Diabetic cardiac autonomic neuropathy, inflammation and cardiovascular disease. J Diabetes Investig 4: 4-18.

4. Pop-Busui R, Boulton AJM, Feldman EL, Bril V, Freeman R, et al. (2017) Diabetic neuropathy: A position statement by the American Diabetes Association. Diabetes Care 40: 136-154

5. Serhiyenko VA, Serhiyenko AA (2015) Diabetic cardiac autonomic neuropathy: Do we have any treatment perspectives? World J Diabetes 6: 245-258.

6. Serhiyenko VA, Serhiyenko AA (2018) Cardiac autonomic neuropathy: Risk factors diagnosis and treatment. World J Diabetes 9: 1-24.

7. Serhiyenko, VA, Serhiyenko AA (2019) Diabetic cardiac autonomic neuropathy. In Saldaña JR (Ed) Diabetes Textbook: Clinical principles, patient management and public health issues. Basel: Springer Nature Switzerland AG pp. 825-850.

8. American Diabetes Association's Standards of Medical Care in Diabetes-2018 (2018) Diabetes Care 42: S1-S194.

9. Bonafini S, Antoniazzi F, Maffeis C, Minuz P, Fava C (2015) Beneficial effects of omega-3 PUFA in children on cardiovascular risk factors during childhood and adolescence. Prostaglandins Other Lipid Mediat 120: 72-79.

10. Tandon N, Ali MK, Narayan KM (2012) Pharmacologic prevention of microvascular and macrovascular complications in diabetes mellitus: implications of the results of recent clinical trials in type 2 diabetes. Am J Cardiovasc Drugs 12: 7-22.

11. Deo SH, Fisher JP, Vianna LC, Kim A, Chockalingam A, et al. (2012). Statin therapy lowers muscle sympathetic nerve activity and oxidative stress in patients with heart failure. Am J Physiol Heart Circ Physiol 303: H377-H385.

12. Moreira ED, Mostarda CT, Moraes-Silva IC, Ferreira JB, dos Santos F, et al. (2013) Effect of simvastatin in the autonomic system is dependent on the increased gain/ sensitivity of the baroreceptors. Physiol Rep 1: e00045.

13. Ramos R, Comas-Cufi M, Marti-Lluch R, Balló E, Ponjoan A, al. (2018) Statins for primary prevention of cardiovascular events and mortality in old and very old adults with and without type 2 diabetes: retrospective cohort study. BMJ 362: k3359.

14. Jeppesen C, Schiller K, Schulze MB (2013) Omega-3 and omega-6 fatty acids and type 2 diabetes. Curr Diab Rep 13: 279-288. [Crossref]

15. Ziegler D, Keller J, Maier C, Pannek J, German Diabetes Association (2014) Diabetic neuropathy. Exp Clin Endocrinol Diabetes 122: 406-415.

16. Simental-Mendia LE, Rodriguez-Moran M, Guerrero-Romero F (2008) The product of fasting glucose and triglycerides as surrogate for identifying insulin resistance in apparently healthy subjects. Metab Syndr Relat Disord 6: 299-304. 
17. Vasques AC, Novaes FS, de Oliveira Mda S, Souza JR, Yamanaka A, et al. (2011) TyG index performs better than HOMA in a Brazilian population: a hyperglycemic clamp validated study. Diabetes Res Clin Pract 93: e98-e100.

18. Abbasi F, Reaven GM (2011) Comparison of two methods using plasma triglyceride concentration as a surrogate estimate of insulin action in nondiabetic subjects: triglycerides $\mathrm{x}$ glucose versus triglyceride/high-density lipoprotein cholesterol. Metabolism 60: 1673-1676.

19. Matthews DR, Hosker JP, Rudenski AS, Naylor BA, Treacher DF, et al. (1985) Homeostasis model assessment: insulin resistance and beta-cell function from fasting plasma glucose and insulin concentrations in man. Diabetologia 28: 412-419.

20. Pursnani A, Massaro JM, D’Agostino RB Sr, O’Donnell CJ, Hoffmann U (2015) Guideline-based statin eligibility, coronary artery calcification, and cardiovascular events. JAMA 314: 134-141.

21. Elnaem MH, Mohamed MHN, Huri HZ, Azarisman SM, Elkalmi RM (2017) Statin therapy prescribing for patients with type 2 dMellitus: A review of current evidence and challenges. $J$ Pharm Bioallied Sci 9: 80-87.

22. Grundy SM, Stone NJ, Bailey AL, Beam C, Kim K, et al. (2018) 2018 AHA/ACC/ AACVPR/AAPA/ACPM/ADA/AGS/AphA/ASPC/NLA/PCNA Guidline on the Management of Blood Chjlesterol. A Report of the American College of Cardiology/ American Heart Association Task Force on Clinical Practice Guidelines. JACC pp. 1-121.

23. Aung T, Halsey J (2018) Associations of Omega-3 Fatty Acid Supplement Use With Cardiovascular Disease Risks: Meta-analysis of 10 Trials Involving 77917 Individuals. JAMA Cardiol 3: 225-234. [Crossref]

24. Poreba M, Mostowik M, Siniarski A, Golebiowska-Wiatrak R, Malinowski KP, et al. (2017) Treatment with high-dose n-3PUFAs has no effect on platelet function, coagulation, metabolic status or inflammation in patients with atherosclerosis and type 2 diabetes. Cardiovasc Diabetol 16: 50.

25. Serhiyenko VA, Serhiyenko LM, Serhiyenko AA (2017) Omega-3 polyunsaturated fatty acids in the treatment of diabetic cardiovascular autonomic neuropathy: A review. In: Moore Sj (Ed) Omega-3: Dietary sources, biochemistry and impact on human health. Nova Science Publishers, New York pp. 79-154.
26. O'Mahoney LL, Matu J, Price OJ, Birch KM (2018) Omega-3 polyunsaturated fatty acids favourably modulate cardiometabolic biomarkers in type 2 diabetes: a metaanalysis and meta-regression of randomized controlled trials. Cardiovasc Diabetol 17: 98. [Crossref]

27. Sawada T, Tsubata H, Hashimoto N, Takabe M, Miyata T, et al. (2016) Effects of 6-month eicosapentaenoic acid treatment on postprandial hyperglycemia, hyperlipidemia, insulin secretion ability, and concomitant endothelial dysfunction among newly-diagnosed impaired glucose metabolism patients with coronary artery disease. An open label, single blinded, prospective randomized controlled trial. Cardiovasc Diabetol 15: 121.

28. Jacobo-Cejudo MG, Valdes-Ramos R, Guadarrama-Lopez AL, Pardo-Morales RV, Martinez-Carrillo BE, et al. (2017) Effect of n-3 polyunsaturated fatty acid supplementation on metabolic and inflammatory biomarkers in type 2 diabetes mellitus patients. Nutrients 9: E573.

29. Kim CH, Han KA, Yu J, Lee SH, Jeon HK, et al. (2018) Efficacy and safety of adding omega-3 fatty acids in statin-treated patients with residual hypertriglyceridemia: ROMANTIC (Rosuvastatin-OMAcor iN residual hyperTrIglyCeridemia), a randomized, double-blind, and placebo-controlled trial. Clin Ther 40: 83-94.

30. Handelsman Y, Shapiro MD (2017) Triglycerides, atherosclerosis, and cardiovascular outcome studies: focus on omega-3 fatty acids. Endocr Pract 23: 100-112.

31. Tenenbaum A, Enrique Z, Fisman EZ (2018) Omega-3 polyunsaturated fatty acids supplementation in patients with diabetes and cardiovascular disease risk: does dose really matter? Cardiovasc Diabetol 17: 119.

32. Calder PC (2015) Marine omega-3 fatty acids and inflammatory processes: Effects, mechanisms and clinical relevance. Biochim Biophis Acta 1851: 469-484.

33. Endo J, Arita M (2016) Cardioprotective mechanism of omega-3 polyunsaturated fatty acids. J Cardiol 67: 22-27.

34. Yanai H, Masui Y, Katsuyama H, Adachi H, Kawaguchi A, et al. (2018) An Improvement of Cardiovascular Risk Factors by Omega-3 Polyunsaturated Fatty Acids. J Clin Med Res 10: 281-289. [Crossref]

Copyright: (C2019 Serhiyenko VA. This is an open-access article distributed under the terms of the Creative Commons Attribution License, which permits unrestricted use, distribution, and reproduction in any medium, provided the original author and source are credited. 